ARQGA / 922

\title{
A VIDEOFLUOROSCOPIA E A QUANTIFICAÇÃO VIRTUAL DO ESVAZIAMENTO GÁSTRICO
}

DESCRITORES - Fluoroscopia. Esvaziamento gástrico.

Os métodos de estudo do esvaziamento gástrico $(\mathrm{Eg})$ têm progredido intensamente nos últimos anos. Desde que o principal desiderato é quantificar os eventos motores desencadeados pela ingestão da refeição, inúmeras aproximações têm sido idealizadas, sempre visando a discriminação dos vários graus de prejuízo da motilidade gástrica. Na maioria das instâncias médicas, trata-se de comparar o Eg de pacientes com o padrão observado em um dos métodos escolhidos. Em geral estes métodos prestam-se suficientemente para diferenciar as alterações mais expressivas do Eg e incluem recursos de imagem radiológica, ultra-sonográfica ou radioisotópica. A precariedade dos dados que se adquirem com eles devem-se à natureza bidimensional das medidas projetadas em áreas de imagem e à natureza discreta das medições em relação ao tempo.

Para comparações mais sutis, como em pesquisa clínica ou experimental, a obsolescência desses estudos tornou-se flagrante. O problema só se resolveu com a medida tridimensional da fração remanescente no estômago da refeição utilizada como teste e com a monitorização contínua do seu decaimento volumétrico. A dupla colimação de refeições marcadas com radiofármacos resolveu de modo muito dispendioso estes problemas. Permitiu, também, a criação de modelos matemáticos de estimativa virtual do Eg e da distribuição volumétrica intragástrica da refeição de prova.

Estes dois parâmetros estão, como agora sabemos, fisiopatologicamente indissociados na compreensão de dispepsias funcionais do tubo digestório alto. Estes modelos, aplicados aos dados adquiridos em duas dimensões e de forma discreta, geraram números e curvas de decaimento do conteúdo gástrico irrealísticos que, se servem para uma aproximação grosseira do Eg, não se prestam para discriminar pequenas diferenças. Isto porque as medidas são também irreais na ausência da captação do espaço de distribuição intragástrico ocupado em dado momento do Eg. Está claro que, estimar tridimensionalmente, de forma inócua e pouco dispendiosa, tornou-se atraente para o estudo do Eg.

A videofluoroscopia agrega a medidas bidimensionais, modelos em que a densidade serve de parâmetro para a terceira dimensão. Por meio de modelagem, expressa por "macros" eletrônicos, permite que se obtenham valores volumétricos em áreas de interesse gástricas sob estudo. Ainda são valores virtuais, porém como FIRMAN et al. (1) $^{(1)}$ demonstraram neste número da Arquivos de Gastroenterologia, podem, gerar uma curva de esvaziamento com eficiência discriminatória, porque são obtidos a intervalos de tempo muito pequenos e podem, também, estimar volumes gástricos regionais ou seja, caracterizar a distribuição intragástrica da refeição remanescente. $\mathrm{O}$ quanto este método permitirá aproximar-se das medidas reais e como se fazer a sua validação para comparações entre grupos nos valores extremos da normalidade, são questões em aberto merecedoras de pesquisa clínica e experimental.

Fábio Pinatel LOPASSO*

\footnotetext{
* Livre Docente de Cirurgia da Faculdade de Medicina da Universidade de São Paulo.
} 
Lopasso FP. The videofluoroscopy and the virtual quantification analysis of gastric emptying. Arq Gastroenterol 2000;37(2): 79 -80.

HEADINGS - Videofluoroscopy. Gastric emptying.

\section{REFERÊNCIA BIBLIOGRÁFICA}

1. Firman CMG, Costa MMB, Costa ML, Lemme E. Avaliação qualitativa e quantitativa do esvaziamento gástrico através do método videofluoroscópico. Arq Gastroenterol, 2000;37:81-8.

\section{BIBLIOGRAFIA RECOMENDADA}

Berstad A, Hausken T Glija OH, Thune N, Matre K, Odegaard S. Volume measurements of gastric antrum by 3-D ultrasonography and flow measurements through the pylorus by duplex technique. Dig Dis Sci 1994;39:97S-100S.

Brown B, Schulze-Delrieu K, Schrier JE. The configuration of the human gastroduodenal junction in the separate emptying of liquids and solids. Gastroenterology 1993;105:433-40.
Parkman HP, Harris AD, Krevsky B, Urbain JL, Maurer AH, Fisher RS. Gastroduodenal motility and dysmotility: an update on techniques avaliable for evaluation. Am J Gastroenterol 1995;90:869-92.

Perkel M, Fajman WA, Hersh T, Moore C, Davidson ED, Haun C. Comparison of the barium test meal and the gamma camera scanning technique in measuring gastric emptying. South Med J 1981;74:1065-8.

Tougas G, Anvari M, Dent J, Somers S, Richards D, Stevenson GW. Relation of pyloric motility to pyloric opening and closure in healthy subjects. Gut 1992;33:466-71. 